\title{
Comment les villageois nomment-ils et s'approprient-ils leurs espaces ressources ? Description d'un socio-écosystème en pays Yambassa, Cameroun
}

\author{
Pauline Gillet $^{1, *}$, Elisabet Codina Llavina ${ }^{1}$, Henri Yambene ${ }^{2}$ et Cédric Vermeulen ${ }^{1}$ \\ ${ }^{1}$ Département BIOSE, Axe de Gestion des Ressources Forestières, Gembloux Agro-Bio Tech, Université de Liège, \\ Passage des Déportés 2, 5030 Gembloux, Belgique \\ 2 Centre National d'Éducation, B.P. 1857, Yaoundé, Cameroun
}

\begin{abstract}
Résumé - La compréhension des rapports qu'une société humaine entretient avec son environnement permet d'anticiper les blocages aux actions de développement rural envisagées, grâce à la perception des mécanismes locaux qui régissent l'accès à la terre et aux ressources. Cette étude décrit les relations entre l'homme et les espaces-ressources, la typologie de l'espace et l'occupation du sol dans un village densément peuplé $\left(65\right.$ habitants $\left./ \mathrm{km}^{2}\right)$ situé à $100 \mathrm{~km}$ au nord de Yaoundé et composé de ressortissants du sous-groupe ethnique Gunu. Les maîtrises foncières exercées sont fortes et vont jusqu'à la reconnaissance administrative de la propriété privée. Toute relance cacaoyère se fera dans le cadre d'espaces fortement disputés, et sans doute au détriment des derniers lambeaux de formations naturelles avec un effet important sur la répartition du travail entre les hommes et les femmes et sur l'appropriation foncière des espacesressources.
\end{abstract}

\begin{abstract}
Mots clés : interface forêt savane / agriculture / occupation foncière / terminologie / canton Gunu-Sud / relance cacaoyère

Abstract - How do the villagers call and appropriate their resource spaces? Description of social ecological system in Yambassa country, Cameroon. The comprehension of the relations that human society have with their environment helps to anticipate the obstacles to rural development measures planned thanks to the comprehension of local mechanisms governing access to land and resources. This study describes the land tenure, typology of space and land uses in a densely populated village ( 65 inhabitants $\left./ \mathrm{km}^{2}\right)$ located $100 \mathrm{~km}$ north of Yaoundé and populated by citizens of the ethnic subgroup Gunu. The land tenures are strong and go up to the official recognition of private property. Any relaunch of the cocoa sector will take place in highly contested areas, and perhaps at the expense of the last remnants of natural formations with a major effect on gender division of labor and land appropriation.
\end{abstract}

Keywords: interface between forest and savannah / agriculture / land occupation / terminology / Gunu-Sud district / relaunch of the cocoa sector

\section{Introduction}

Les sociétés humaines dont l'économie est basée sur l'utilisation intensive du territoire local sont susceptibles d'entraîner des modifications d'utilisation de la terre au sein de ce territoire. Ces changements sont aussi liés aux adaptations des usages et à l'ouverture des marchés à plus

\footnotetext{
*Auteur de correspondance : pgillet@ulg.ac.be
}

grande échelle (Gillet et al., 2015 ; Sonter et al., 2015). En Afrique centrale, l'étude de ces sociétés est considérée comme prioritaire, et les interventions de développement rural les visent particulièrement, principalement pour proposer une augmentation de la production agricole (Magne et al., 2014 ; Pédelahore, 2014). Pourtant, toute intervention de développement rural devrait être précédée de la connaissance du système de production et des rapports que la société humaine concernée entretient avec son environnement. Le concept de socio-écosystème, entendu comme le résultat d'une coévolution entre économie, culture, technologie, écologie 
et développement institutionnel à différentes échelles (Maertens et Rotmans, 2005) permet l'utilisation d'un langage conjoint entre sciences sociales et sciences de l'environnement dans le but de dépasser les prescriptions réductrices habituellement proposées dans le cadre de politiques d'aide au développement et de développement rural (Ostrom et Cox, 2010). Cette connaissance passe entre autres par l'étude de l'espace vécu, des représentations et des typologies locales de l'espace que la population développe par rapport au milieu où elle évolue (Vermeulen et al., 2008). Quelques auteurs ont étudié les typologies de différentes ethnies forestières d'Afrique centrale (Alexandre et Binet, 1958 ; De Wachter, 2001 ; Vermeulen et Carrière, 2001 ; Vermeulen et al., 2008), mais la plupart concernent des groupes d'essarteurs inscrits dans des environnements de forêt dense, avec un environnement relativement intact, des densités de population faibles et des socio-écosystèmes en mutation lente. Les études portant sur la caractérisation de socio-écosystèmes en mutation rapide, dans un environnement très anthropisé, avec une densité de population élevée sont, elles, très rares, alors que ce type de socio-écosystème se multiplie autour des capitales d'Afrique centrale. Notre étude porte sur un village situé dans l'arrondissement de Bokito, lequel relève du canton GunuSud en pays Yambassa au Cameroun (classé sur le plan linguistique par Guthrie [Robinson, 1983] et Paul et al., 2015 en A.622, 639-3 yas). Le village est confronté à des changements sociaux et fonciers rapides liés à la dynamique de la proche capitale Yaoundé qui permet des échanges commerciaux importants. La production vivrière et la culture cacaoyère, principales sources de revenus, y rencontrent de nombreux problèmes (Somwag, 2014). Des solutions issues du Ministère de l'agriculture y sont envisagées, dans un contexte de prix des marchés mondiaux favorables à la cacaoculture et de relance cacaoyère (Jagoret et al., 2014). Il se pose donc pour ce socio-écosystème des questions cruciales : quels seront les effets d'une relance cacaoyère dans la zone sur l'évolution de ce dernier? Quelles seront les conséquences sur les maittrises foncières ? Peut-on envisager les effets collatéraux d'une politique tournée vers les cultures de rente ?

L'objectif de cette étude est la description du socioécosystème, à travers la description des typologies, des représentations locales et du vocabulaire lié aux ressources naturelles chez les Gunu-sud afin d'identifier les maîtrises foncières que ces notions recouvrent, informations cruciales à obtenir avant d'envisager une quelconque intervention. Ces maîtrises seront ensuite structurées selon la théorie des maîtrises foncières développée par Le Roy et al. (1996). Cette théorie a été développée pour structurer les politiques de conservation et de gestion sur la base des concepts fonciers traditionnels. Elle permet de rendre compte du mode africain d'organisation du foncier en se référant à des termes occidentaux utilisés pour qualifier le droit de propriété (Le Roy, 1995). En effet, en Afrique centrale, l'occupation spatiale est légitime, il existe peu de propriété foncière. La théorie des maîtrises foncières exprime des degrés de contrôle exercé sur des biens ou des lieux (identifiés comme les objets de maîtrise) et permet d'identifier les relations entre les hommes exerçant ces maitrises (les sujets de maîtrise). Elle illustre la diversité des rapports exercés ainsi que leurs nuances, plus étendues que les classiques oppositions entre privé et public issus de la logique occidentale.

\section{Matériels et méthodes}

\subsection{Contexte général}

Le pays Yambassa est constitué de 36 villages regroupés en cinq cantons coutumiers (Elip, Gunu-nord, Gunu-sud, Mmala et Yangben) et se situe au centre du Cameroun, à $100 \mathrm{~km}$ au nord-ouest de la capitale Yaoundé, dans la vallée de la Lebomo, affluent du fleuve Sanaga (Yambene Bomono, 2012a). La végétation se situe à l'interface entre forêts et savanes : le paysage est constitué de savanes parsemées de forêts galeries le long des cours d'eau ainsi que de forêts issues des plantations humaines. La présente étude se focalise sur un village du canton Gunu-sud localisé dans l'arrondissement de Bokito (Fig. 1).

\subsection{Méthodologie}

L'acquisition des informations a eu lieu dans le cadre du projet CoForTips (CIRAD, 2015) de février à mai 2014. Elle a débuté par la mise en place d'un recensement total de la population $(100 \%$ des ménages d'une population de 455 ménages). Ce recensement a été suivi d'observations participantes et d'enquêtes semi-directives individuelles (auprès de 30 femmes et hommes agriculteurs choisis aléatoirement) ou en groupe-cible afin de comprendre les termes liés à l'utilisation de l'espace. Des réunions collectives ont servi à croiser et confirmer les informations obtenues (Le Meur, 2002). De plus, la cartographie participative a permis de définir les activités villageoises et de comprendre leur répartition dans le finage villageois (Larzillière et al., 2013). Les activités ont ensuite été localisées à l'aide de récepteurs GPS lors de déplacements sur les lieux avec les villageois afin de représenter cartographiquement les indicateurs d'occupation spatiale tels que les zones de champs, de chasse, de pêche et de collectes, les sources et les lieux-dits (Vermeulen et al., 2011). Cette étape a également permis de mesurer l'étendue du finage, du terroir, et la surface moyenne utilisée par ménage cultivateur (mesurée à partir des champs de 11 ménages d'âges variés et auprès de 10 ménages propriétaires d'une cacaoyère), ainsi que de dresser une carte de l'occupation spatiale actuelle. Dans cette étude nous entendons par finage l'espace dans lequel la communauté exerce ses droits d'usages (Le Roy et al., 1996) et par terroir l'ensemble des terres agricoles soumis au cycle cultural au sein du finage (Le Roy et al., 1996). Les termes Gunu utilisés sont orthographiés d'après le code A.622, 639-3 yas (Paul et al., 2015). Toutes ces informations ont par la suite été présentées aux villageois lors d'une réunion de restitution permettant de valider et d'affiner les renseignements collectés au cours des quatre mois d'enquêtes.

\section{Résultats}

\subsection{Description de la population du village}

Les résultats du recensement ainsi que de l'étude de l'occupation spatiale du village indiquent une population de 2,560 habitants permanents pour une surface de finage de 3,959 ha, soit une densité de population de 64,7 habitants $/ \mathrm{km}^{2}$, lesquels habitants pratiquent majoritairement l'agriculture. Cette densité est plus élevée que la moyenne nationale 

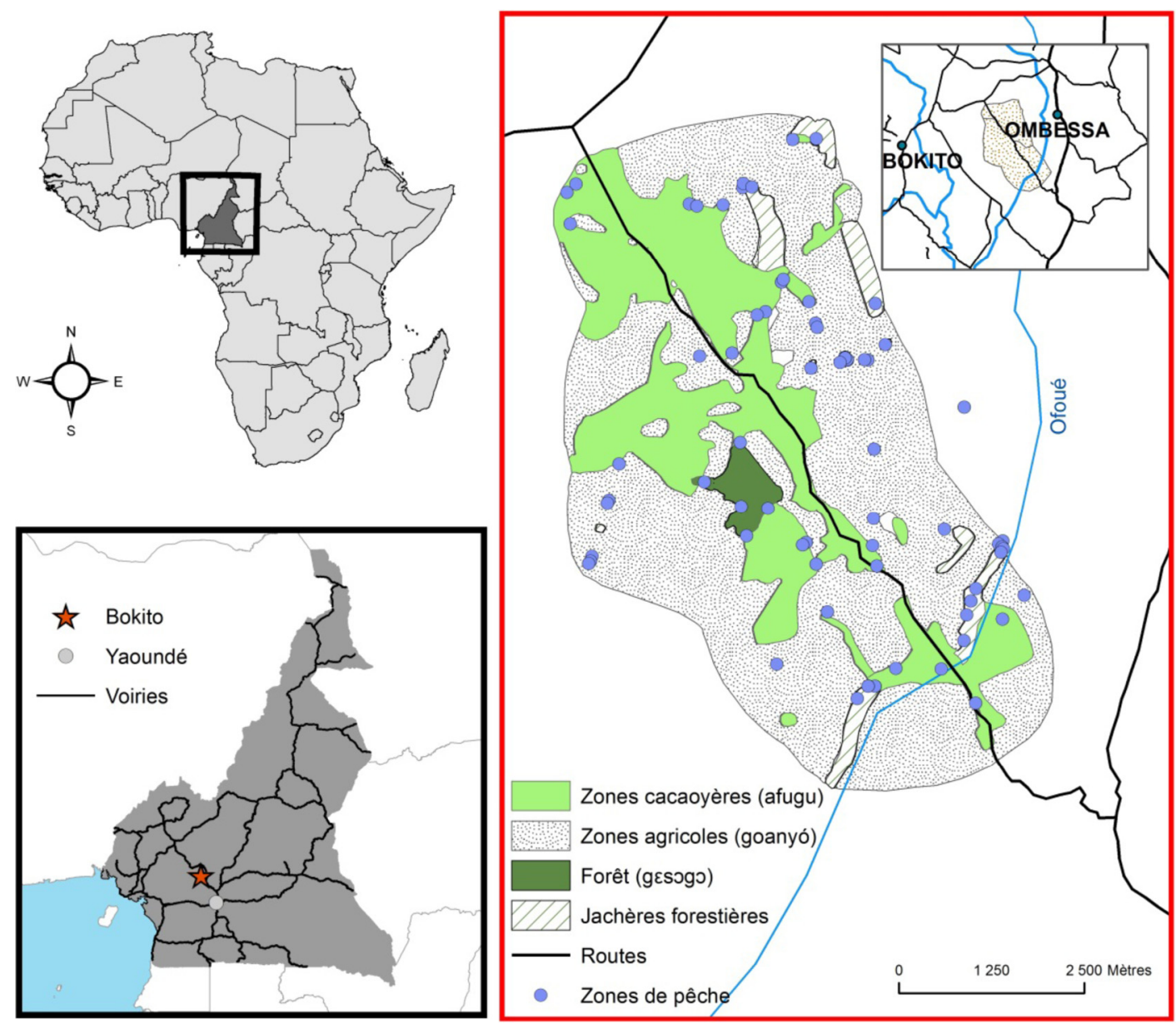

Fig. 1. Localisation du finage villageois en pays Gunu et répartition des zones agroforestières et agricoles.

Fig. 1. Location of village's territory and distribution of agro-forestry and agricultural areas.

(49,9 habitants $/ \mathrm{km}^{2}$ en 2015 [Statistiques mondiales, 2015]) et importante dans le contexte du bassin du Congo. La mixité ethnolinguistique de la population est très faible. En effet, $93 \%$ de la population est issue du groupe Gunu. Les $7 \%$ restants sont majoritairement représentés par des femmes issues des villages environnants ayant épousé des hommes du village. Ce résultat illustre les propos de Filipski et al. (2007) relatant que contrairement à d'autres situations en Afrique centrale, le pays Yambassa en général ne subit qu'une faible pression d'immigration. Le village étudié est constitué de différents quartiers correspondant aux clans rassemblés par la colonisation autour des années 1890 dans le but de faciliter la gestion administrative (Jagoret, 2011).

\subsection{La diversité des espaces et des ressources traduite par la richesse du vocabulaire Gunu}

La liste du vocabulaire Gunu lié aux espaces-ressources est dressée dans le Tableau 1. Sa richesse montre l'importance de ces espaces-ressources dans le système de production. Le nombre élevé de termes liés aux différents espaces de cultures confirme la place prépondérante de l'agriculture dans le système de production actuel.
Chez les Gunu, il existe une nette division entre la savane (goanyó) et la forêt (gesıgo) au sein de l'espace du village

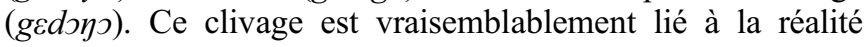
physique de l'environnement mais il est aussi historique. En effet, la forêt était jadis fortement recherchée afin de s'abriter des ennemis et est associée aux ancêtres y ayant vécu, alors que la savane évoquerait l'insécurité liée aux conflits et aux grands mammifères (Yambene Bomono, 2012a). Les habitants se sont approprié et ont exploité ces zones, en maintenant cette dichotomie entre zone forestière et zone savanicole. La forêt « spontanée » $(g \varepsilon b \supset n d \varepsilon)$ constitue la source principale des produits forestiers ligneux utilisés pour la construction, et l'origine de la plupart des produits non ligneux entrant dans la composition de l'alimentation et de la pharmacopée. Cette forêt constitue aussi l'endroit idéal pour l'installation de plantations cacaoyères (afugu). Dans le terroir villageois étudié, les plantations cacaoyères ont saturé l'espace forestier existant, ne laissant indemnes que de petites surfaces de forêts sacrées. La plantation de cacaoyers en savane a augmenté de façon importante la surface arborée dans la zone (Yambene Bomono, 2012b), favorisant la récolte de produits forestiers non ligneux et l'installation d'espèces forestières indigènes. On observe en effet une augmentation de la surface boisée de 5,8\% entre 1990 et 2013 dans le village (Oszwald et al., 2015). 
Tableau 1. Typologie de l'espace vécu en Gunu.

Table 1. Typology of space in Gunu.

\begin{tabular}{|c|c|}
\hline Espace vécu & Nom en Gunu \\
\hline Village & tésวnว, gعdวฤว \\
\hline Quartiers, portion du territoire & عfágáfágaá \\
\hline Limite & ombálá \\
\hline Piste & 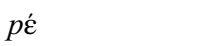 \\
\hline Route & gebala \\
\hline Clans & boya \\
\hline
\end{tabular}

Forêt et savane

\begin{tabular}{|c|c|}
\hline Forêt & gesวgว \\
\hline Forêt « spontanée » & gebวnde \\
\hline Petits bosquets & idúne \\
\hline Savane & goanyó \\
\hline Forêts marécageuses & odóbó \\
\hline Limite forêt-savane & buaya bagesog \\
\hline
\end{tabular}

Champs, plantations et jachères

\begin{tabular}{|c|c|}
\hline Savane commune & kวnds \\
\hline Savane de bas-fonds avec eau en permanence & geวye \\
\hline Savane de bas-fonds avec eau en saison des pluies & osćbé \\
\hline Champs & $n t \varepsilon ́ m \varepsilon ́$ \\
\hline Plantation & afugu \\
\hline Jachères & bifúgú \\
\hline Nouveau champ & ncée \\
\hline Chemin de délimitation des champs & buaya ba ntéme \\
\hline
\end{tabular}

Espace de chasse
Chasse en général

Piéger

Ensemble de pièges (pour une

personne, mais à plusieurs

endroits)

Petit piège (traditionnel)

Pièges pour les grands

mammifères

Chasse au fusil

Chasse à l'arc

Chasse à la fronde

Chasse de nuit

Gibier

Espace, techniques et produits de pêche

\begin{tabular}{|c|c|}
\hline Eau & miimpo \\
\hline Puits & nobéláa \\
\hline Source ou étang naturel & osวmbっ \\
\hline Source ou étang aménagé & gecóç \\
\hline Rivières & nofámbálá \\
\hline Lacs naturels & guiigi \\
\hline Étangs artificiels & osวmbว et gecócs \\
\hline Pêche & gorta \\
\hline
\end{tabular}

Chasse collective
Tableau 1. (suite).

Table 1. (continued).

Espace, techniques et produits de pêche

Pêche au barrage

Pêche à la nivrée

Pêche à la canne

Pêche à la nasse

Poissons

Poisson d'eau douce
Les Gunu considèrent trois zones de savane en fonction de la rapidité d'évacuation de l'eau : la savane typique ( $k \supset n d s)$ où l'eau ne s'accumule jamais, la savane de bas-fond où l'eau s'accumule en permanence ( $g \varepsilon \supset y \varepsilon$ ) et la savane de bas-fond d'où l'eau s'évacue plus rapidement (osćbé). Chaque zone et ses caractéristiques conditionnent les espèces cultivées ainsi que la durée de jachère pratiquée entre deux périodes de culture (Fig. 2).

La pratique actuelle de la chasse ne permet plus que la capture de petits rongeurs, inféodés au milieu rural (Gillet et al., 2015). Malgré la modestie du tableau de chasse, plusieurs techniques sont employées et cette activité traditionnelle est encore pratiquée par de nombreux villageois. Des lignes de pièges (bédámba) sont fréquemment posées aux abords des champs, il s'agit de pièges « à cou » (padegena) ou de pièges à appâts. La chasse à l'aide de lances, d'arcs (gipuimé gofetó) ou de fusils (gipuimé ga mamba), et des chasses collectives à l'aide de chiens (gipuimé gatano) sont organisées dans les zones boisées. Enfin, le recours aux feux de brousse en fin de saison sèche est fréquent pour déloger le gibier (niámá) de la savane et le concentrer dans les bosquets (Yambene Bomono, 2010). Ces feux échappent régulièrement à la vigilance des chasseurs, occasionnant des dégâts importants aux cultures, plantations et même aux habitations.

Tout comme le gibier, la diversité, la quantité et la taille des poissons semblent avoir, à dire d'acteurs, fortement diminué ces dernières décennies. Si le vocabulaire relatif à la pêche paraît riche, les villageois ne pratiquent pourtant plus aujourd'hui qu'une pêche très limitée. Les ressources halieutiques épuisées, certaines personnes se sont approprié des zones de bas-fonds forestiers marécageuses (odóbó) et y ont creusé des étangs (oşmbo) dans le but d'y pratiquer la pisciculture.

\subsection{Occupation de l'espace}

Toutes ces notions de vocabulaire ne donnent qu'une vue partielle de la façon dont les Gunu occupent l'espace. La Figure 2 en donne une représentation spatiale. Dans le village étudié, le finage s'étend sur environ 3,950 hectares et le terroir occupe 94,6 \% de la surface du finage. La majorité de l'espace $(66,6 \%)$ est consacrée à l'agriculture destinée tant à la consommation familiale qu'à la vente. Il s'agit donc d'un village dont l'environnement est très fortement anthropisé.

Même si l'homme est actif dans les travaux agricoles pour les activités nécessitant une force physique importante, 


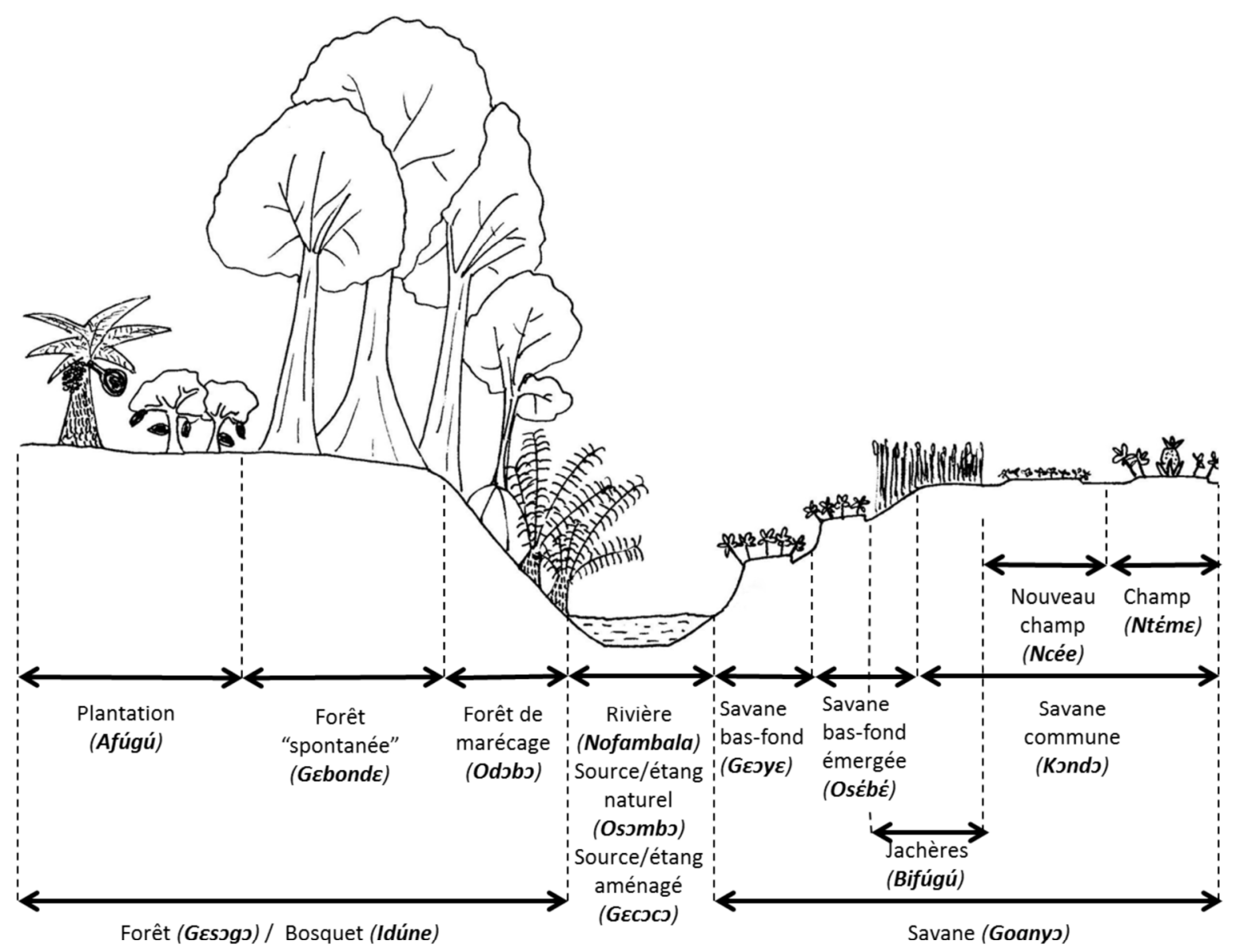

Fig. 2. Dénomination du type de champs dans les zones agroforestières et agricoles en fonction de la situation topographique en pays Gunu. Fig. 2. Field type designation depending on the topographic situation.

l'agriculture vivrière est principalement le fait des femmes. Elle est pratiquée en savane sur une superficie moyenne de 1,4 ha par femme cultivatrice, valeur à comparer par exemple aux 0,5 ha par femme cultivatrice observés chez les Badjoué en forêt dense de l'Est Cameroun (De Wachter, 2001). Cette surface est divisée en parcelles surélevées de 30 à 40 centimètres pour permettre l'évacuation de l'eau. Dans le $k$ ondo, les champs ont une surface moyenne de 0,14 ha $( \pm 0,08 \mathrm{ha})$, chaque femme en cultive une dizaine pour une surface de 1,37 ha $( \pm 0,29 \mathrm{ha})$ par femme cultivatrice. Les produits cultivés sont très diversifiés (entre autres manioc, ignames, arachides, maïs, plantain et papaye), la culture dure de 3 à 20 ans. Dans un système agricole sans intrants, la restauration de la fertilité est assurée par le retour de la jachère (Carrière, 1999). Celle-ci dure de 3 à 10 ans. Dans le gesyz et l'osébé, les champs sont appelés des "buttes », mesurent $54 \mathrm{~m}^{2}\left( \pm 5,7 \mathrm{~m}^{2}\right)$ et sont surélevés de 50 à $70 \mathrm{~cm}$. Les femmes exploitent chacune en moyenne $14( \pm 6,6)$ buttes pour une superficie de 0,07 ha ( $\pm 0,05$ ha). On y cultive essentiellement du manioc et du taro pendant une année suivie de cinq ans de jachères.

La surface agroforestière villageoise est d'environ $12 \mathrm{~km}^{2}$ (environ $30 \%$ du finage total). Elle englobe les cacaoyères, les quelques palmeraies (installées par les élites du village) et les jardins de case. La surface des cacaoyères varie de 0,6 à 3,4 ha. Les cacaoyères sont le plus souvent plantées dans la savane étant donné la rareté des zones boisées sans occupation agroforestière (Yambene Bomono, 2012b). L'absence de couvert forestier nécessite la plantation d'espèces compagnes telles que bananiers, agrumes et autres arbres fruitiers fournissant l'ombrage nécessaire au développement des plantules. Le couvert arboré permet alors le développement d'essences indigènes qui seront conservées sur pied jusqu'à leur maturité en cas de débouchés économiques (bois d'œuvre ou fruitiers indigènes).

\subsection{Niveau de cogestion et maîtrises des espaces- ressources}

L'occupation physique de l'espace s'accompagne de règles foncières et de maîtrises diversifiées sur les espaces-ressources. Chez les Gunu, les différents niveaux de cogestion observés sont :

- les groupes affinitaires sans liens familiaux évidents ;

- les groupes géographiques, le plus souvent issus de quartiers (Efágáfágaá) ;

- les lignages (constitués des descendants d'un ancêtre connu) (Toison, 2008) ;

- la famille au sens large rassemblant les grands-parents et leurs descendants ;

- l'unité familiale au sens strict rassemblant les descendants directs d'une des femmes du fondateur de la famille ;

- l'individu. 
Tableau 2. Régulations possibles des rapports de l'homme à la terre et aux ressources par les maîtrises foncières. Cas des Gunu au Cameroun. Adapté de Le Roy et al. (1996)

Table 2. Possible regulation of human relationships with land and resources by land tenure. Case of Gunu in Cameroun. Adapted from Le Roy et al. (1996)

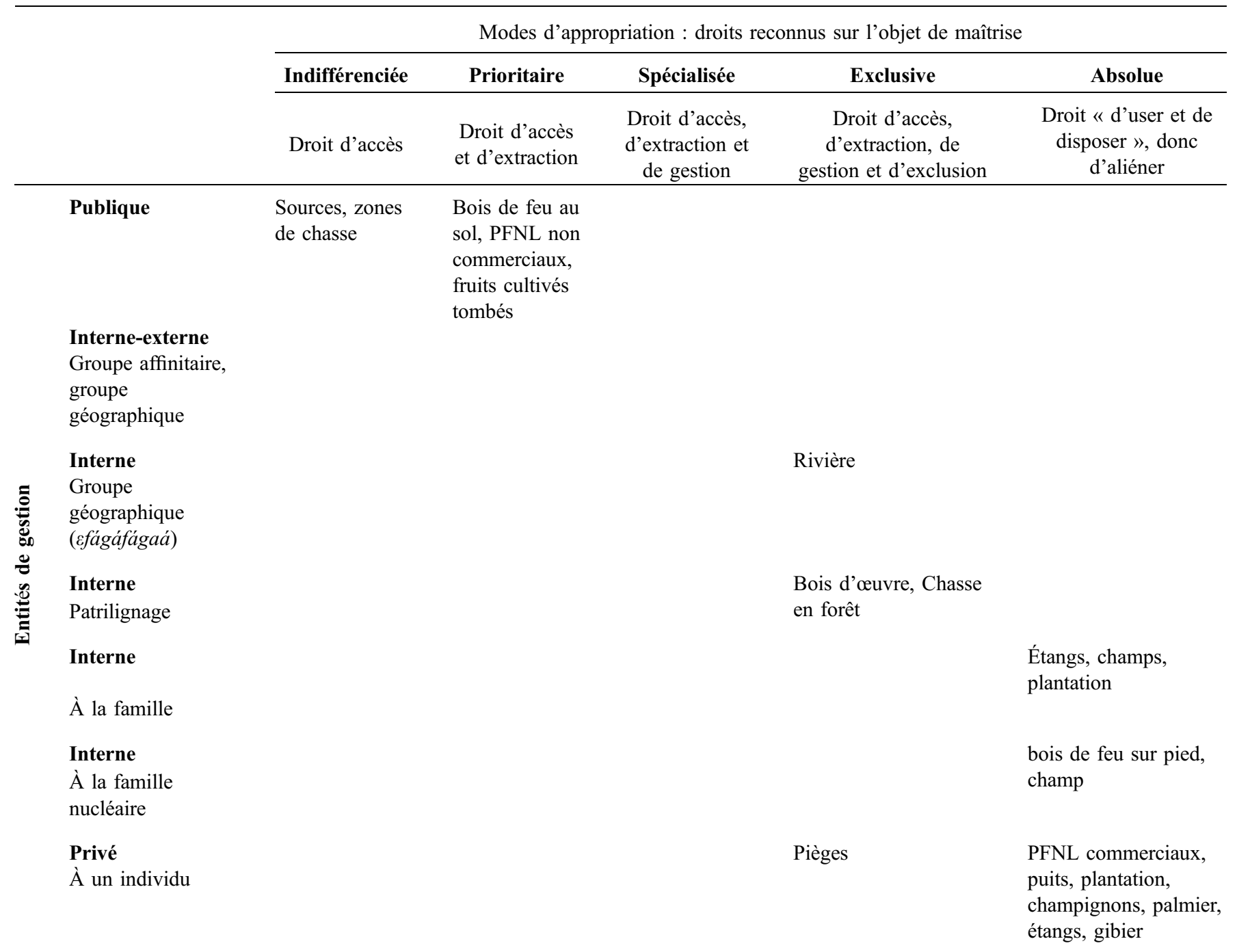

PFNL : Produits forestiers non ligneux.

Les régulations possibles des rapports de l'homme à la terre et aux ressources sont présentées sous forme de tableau (Tab. 2) reprenant verticalement les niveaux de cogestion énoncés cidessus et horizontalement des niveaux d'appropriation, allant de la maîtrise indifférenciée donnant uniquement le droit d'accès, à la maîtrise exclusive et absolue donnant le droit d'aliéner.

On observe au Tableau 2 que l'espace est décliné en plusieurs sous-espaces liés principalement au type d'activité (espace agricole, espace de pêche, espace de chasse et espace de cueillette).

\subsubsection{Espace agricole}

Les maîtrises exercées sur les zones agricoles sont identifiées par les sous-espaces « champs » et « plantations ». Les champs et les espèces cultivées sont localisés dans la partie inférieure droite du Tableau 2. C'est l'unité familiale qui se les approprie assez fortement. D'après les villageois, par le passé, c'est plutôt le clan qui s'appropriait les savanes, puis ensuite les familles, par la défriche et la mise en culture. Selon les acteurs interrogés, la monétarisation, l'effritement des valeurs collectives, l'individualisme grandissant auraient eu raison de cette pratique. Actuellement, toutes les terres ont été accaparées à l'échelon familial ou individuel. L'appropriation foncière est très importante et débouche parfois jusqu'à la reconnaissance administrative de la propriété de la terre. L'obtention de la terre a lieu par héritage, location ou vente. Les plantations comprenant des arbres à bois d'œuvre, des cacaoyers et des arbres fruitiers sont le plus souvent propriété des hommes et font l'objet d'un partage entre les fils lors de l'héritage.

\subsubsection{Espace de chasse et de pêche}

Les maitrises exercées sur les zones de chasse diffèrent en fonction du type d'occupation du sol. Les zones de chasse en 
forêt ( $g \varepsilon s \jmath g \jmath)$ sont réservées à certains patrilignages, les zones de piégeage localisées le plus souvent en dehors de la forêt sont accessibles à tous les Gunu du village, mais les pièges et les produits de la chasse (niámá) sont réservés au propriétaire du piège. Parmi les différents types de pêche, la technique la plus pratiquée est la pêche au barrage (gossa). Les étangs appartiennent toujours à celui qui les a creusés. Ceci implique aussi l'appropriation du petit bosquet (idúne) qui les environne. Ces étangs peuvent être gérés par la famille au sens large ou devenir la propriété d'un seul héritier. Ils font alors l'objet d'une maîtrise privée absolue.

\subsubsection{Espace de cueillette}

Les produits forestiers non ligneux (PFNL) non commerciaux, qu'il s'agisse de produits liés à la pharmacologie traditionnelle ou de fruits, sont accessibles à tous, même dans les champs et les plantations qu'un individu s'est approprié. C'est le cas des escargots (Achatina fulica), des vers blancs du palmier (Gonimbrasia belina et Gynanisa maia), des champignons et du miel. Les plantes productrices de PFNL commerciaux comme le Raphia hookeri, Canarium schweinfurthii, Tetracarpidium conophorum ou Cola pachycarpa, appartiennent au propriétaire du terrain. Les fruits cultivés tombés au sol peuvent aussi être ramassés par tous dans n'importe quelle plantation, mais pas à des fins lucratives. Les palmiers en vie appartiennent à la personne qui possède le terrain cultivé ou en jachère. Les palmiers morts, comme les autres arbres, deviennent publics et sont utilisés par tous les ressortissants du village comme bois de chauffage. Ils ne peuvent être abattus, mais s'ils sont à terre tout le monde peut les exploiter.

\section{Discussion}

\subsection{Comparaison du socio-écosystème étudié à des socio-écosystèmes forestiers d'Afrique centrale}

En comparant le tableau des typologies Gunu en pays Yambassa avec ceux des Kota (Vermeulen et al., 2008) ou des Badjoué (De Wachter, 2001), deux ethnies de forêts denses d'Afrique centrale, on constate que le vocabulaire associé à la forêt et aux pratiques inhérentes à cet environnement est assez pauvre chez les Gunu. Ceci s'explique sans doute autant par la situation initiale, à l'interface de la forêt et de la savane, que par la pression foncière actuelle ayant diminué la disponibilité de ces ressources (Gillet et al., 2015, 2016). Au contraire, le vocabulaire lié à la différenciation des champs et plantations est très étendu, témoignant de l'importance économique des activités agricoles dans la zone. Contrairement aux ethnies forestières (Vermeulen et Carrière, 2001 ; Vermeulen et al., 2008), il n'existe pas de terminologie spécifique aux différents types de jachères et leur appropriation ne diffère pas de celle des champs. Cette absence reflète sans doute la réduction forte du temps de jachère et la disparition de la jachère arborée dans le système de production observée sur le terrain.

Du point de vue de l'occupation spatiale, Vermeulen et al. (2008) signalent que dans le cas d'une communauté vivant en forêt dense, les activités de chasse et de collecte se répartissent souvent le long de quelques pistes principales. Le terroir est inscrit dans un rayon de 2 à 3 kilomètres autour du village. Le finage, de vaste taille (allant de 10000 à 25000 ha pour des villages de 150 à 500 habitants), est soumis à une appropriation dégressive quand on s'éloigne de son centre. Ces observations ne concordent pas avec ce que nous avons observé chez les Gunu, qui se distinguent clairement d'une société vivant en forêt dense. La densité de population élevée du village, la pression foncière importante ainsi que la dépendance économique à la production agricole ont conduit à une course à la terre, entraînant une expansion des activités agricoles dans la presque totalité du finage villageois.

Les niveaux de cogestion ont été identifiés chez plusieurs ethnies du Cameroun et du Gabon forestier : on en dénombre cinq chez les Kota (Vermeulen et al., 2008), sept chez les Ntumu et cinq chez les Badjoué (Vermeulen et Carrière, 2001). $\mathrm{Du}$ fait de leur espace villageois très étendu, ces ethnies forestières n'exercent qu'une maîtrise faible sur leur finage. En raison de son occupation agricole importante et de sa densité de population élevée, la zone étudiée au contraire est sujette dans son ensemble à des maîtrises beaucoup plus fortes sur les espaces-ressources. Les zones de pêche, les plantations et les champs font souvent l'objet d'une appropriation exclusive allant jusqu'à la reconnaissance juridique et privée de la propriété des terrains. Le Roy (1995) prédisait que dans une situation de marchandisation imparfaite de la terre, l'affermissement des maîtrises foncières serait le reflet de la diminution de la quantité de terres disponible par unité familiale pour la pratique de ses activités. Une affirmation que confirme notre cas d'étude.

\subsection{Les effets d'une relance cacaoyère dans le socio-écosystème étudié}

La description des typologies locales et des relations entre l'homme et les espaces-ressources sont des approches utilisées pour permettre la compréhension des socio-écosystèmes et pour structurer des interventions de développement rural. En particulier elles sont fréquentes en cas de projet de mise en place de forêts communautaires. Dans ce cadre, la compréhension des typologies utilisées ainsi que des relations entre l'homme et les espaces ressources permettent de définir les acteurs villageois concernés par ces projets ambitieux ou d'identifier d'autres actions communautaires génératrices de revenus à mettre en place (vergers, exploitations de produits forestiers non ligneux, élevage) (Fankap et al., 2001 ; Marien et al., 2013 ; Vermeulen et al., 2008, 2011, 2015). Dans notre cas d'étude, la connaissance de ces typologies et des relations entre l'homme et les espaces-ressources permettent par exemple de définir de façon raisonnée les espaces d'actions prioritaires ainsi que les acteurs à considérer prioritairement dans le cadre de projets de valorisation économique de l'agriculture ou de relance cacaoyère (Jagoret et al., 2014), dans ce contexte particulier de transition entre des espaces-ressources jadis gérés à l'échelon collectif et aujourd'hui de plus en plus tournés vers une gestion familiale, voire individuelle. Concrètement, ces connaissances nous enseignent que toute relance cacaoyère se fera dans le cadre d'espaces fortement disputés, et sans doute au détriment des derniers lambeaux de formations forestières naturelles et sur des espaces de savane d'ores et déjà inclus dans des rotations jachères-champs vivriers. La suppression de ces 
derniers écosystèmes naturels aurait pour conséquence la diminution des zones de refuge pour les dernières espèces de faune sauvage ainsi que des PFNL associés aux zones forestières, même si de nombreuses espèces fruitières sont conservées en cacaoyères (Jagoret et al., 2009). De plus, la surface du finage villageois étant limitée, l'acquisition de parcelles dans les villages voisins ou dans des zones bien plus éloignées doit être envisagée. Au sein du finage villageois, la cacaoculture pourrait concurrencer la production vivrière en termes de colonisation des zones de champs actuelles, mais aussi en termes de main-d'œuvre. En effet, même si l'agriculture vivrière reste le fait majoritaire des femmes, la main-d'œuvre masculine est nécessaire pour les activités nécessitant une force physique importante comme les travaux d'abattage et de débroussage des jachères de longue durée. Notons toutefois que la main-d'œuvre masculine n'est pas indispensable dans le cas de l'ouverture d'un champ en savane, sans jachère arborée. La diminution de la participation des hommes dans le débroussage pourra entraîner un raccourcissement du temps de jachère et à terme, une diminution importante de la fertilité des sols. Le passage de la culture vivrière à la cacaoculture entraîne de plus un changement de maîtrises peu documentées : les maîtrises du genre. En effet, si les revenus issus des cacaoyers sont principalement gérés par les hommes, les champs vivriers majoritairement cultivés par les femmes font l'objet d'une appropriation interne à la famille et la gestion des revenus dégagés par ces champs permet aux femmes une certaine indépendance économique (Boserup, 1985 ; Feintrenie et al., 2015). En plus de basculer vers une maîtrise privée et exclusive des plantations cacaoyères (Filipski et al., 2007), l'augmentation de l'espace en cacaoyères, si elle se fait aux dépens de l'espace disponible pour la production vivrière, entraînerait la mutation d'une économie villageoise basée sur la production agricole vivrière vers une économie de rente. Cela participerait à une diminution $\mathrm{du}$ potentiel de génération de revenus par les femmes et écarterait ces dernières d'une capacité de production autonome les rendant économiquement dépendantes de leurs époux.

\section{Conclusion}

L'étude des interactions entre l'homme et son environnement ainsi que le vocabulaire qu'une société utilise pour rendre compte des ressources qu'elle exploite constituent le socle d'une description plus complète du socio-écosystème considéré. Dans notre site d'étude, localisé en pays Gunu au Cameroun, la densité de population élevée occasionne une pression foncière importante limitant l'espace disponible pour les activités villageoises. Ces activités génératrices de revenus sont majoritairement axées sur les activités agricoles vivrières et de rente, entraînant une richesse importante du vocabulaire utilisé pour nommer les différents espaces agricoles. La compréhension de ces différentes notions constitue une connaissance préalable indispensable pour structurer une intervention en développement rural selon des actions ciblées aux espaces-ressources identifiés et selon les modalités de cogestion observées. Elle permet d'anticiper les blocages aux actions envisagées, par la compréhension des mécanismes locaux qui régissent l'accès à la terre et aux ressources. En cas de relance cacaoyère dans la zone, il est probable que les derniers écosystèmes naturels soient sacrifiés. De plus, une attention particulière devra être apportée à la concurrence entre produits vivriers et de rente. En plus de l'effet important en termes d'autonomie alimentaire, la disparition des champs vivriers pourrait aussi entraîner une diminution de la génération de revenus par les femmes et écarterait ces dernières d'une capacité de production autonome. Les résultats de cette étude, géographiquement localisée, peuvent être transposés à d'autres zones agricoles présentant une densité démographique importante et une agriculture de rente active.

\section{Références}

Alexandre P, Binet J. 1958. Le groupe di Pahouin (Fang, Bulu, Beti). In: Monographies ethnologiques africaines de l'institut international africain. Paris: Presse Universitaire de France, pp. 73-76.

Boserup E. 1985. Economic and Demographic Interrelationships in sub-Saharan Africa. Popul. Dev. Rev. 11 (3): 383

Carrière S. 1999. "Les orphelins de la forêt». Influence de l'agriculture itinérante sur brûlis des Ntumu et des pratiques agricoles associées sur la dynamique forestière du sud Cameroun. Thèse de doctorat. Montpellier II Sciences et Techniques du Languedoc, Montpellier, France.

CIRAD. 2015. Site des projets CoForTips et CoForSet. Retrieved from http://www.cofortips.org/

De Wachter P. 2001. L'agriculture itinérante sur brûlis, base de l'économie Badjoué. In: La forêt des hommes : terroirs villageois en forêt tropicale africaine. Les presses agronomiques de Gembloux, Willy Delvingt, pp. 15-42.

Fankap R, Doucet J-L, Dethier M. 2001. Valorisation des produits forestiers non ligneux en forêt communautaire. In: La forêt des hommes : terroirs villageois en forêt tropicale africaine. Les presses agronomiques de Gembloux. Willy Delvingt, pp. 145-168.

Feintrenie L, Gillet P, Garcia C, et al. 2015. Family farming in a changing landscape: how activities change when forest disappears. In: Annual World Bank Conference on Land and Poverty: "Linking Land Tenure and Use for Shared Prosperity”, Washington DC.

Filipski M, Colin J-P, Seignobos C. 2007. Émergence et évolution des droits de propriété dans un contexte d'abondance foncière. Le cas du pays yambassa (Cameroun). Cah. Agric. 16 (5): 387-393.

Gillet P, Vermeulen C, Doucet J-L., Lehnebach C, Codina E, Feintrenie L. 2016. Deforestation rate has an impact on non-timber forests products in Central Africa. Forests 7 (5).

Gillet P, Vermeulen C, Lehnebach C, Codina Llavinia E. 2015. What do human eat when forests disappear. Nat. \& Faune 29 (2): 34-36.

Jagoret P. 2011. Analyse et évaluation de systèmes agroforestiers complexes sur le long terme : application aux systèmes de culture à base de cacaoyer au Centre Cameroun. Thèse de doctorat. Montpellier SupAgro, Montpellier.

Jagoret P, Bouambi E, Ngogue H, Battini J-L, Nyassé S. 2009. Diversification des exploitations agricoles à base de cacaoyer au Centre Cameroun : mythe ou réalité ? Biotechnol. Agron. Soc. Environ. 13 (2): 271-280.

Jagoret P, Kwesseu J, Messie C, Michel-Dounias I, Malézieux E. 2014. Farmers' assessment of the use value of agrobiodiversity in complex cocoa agroforestry systems in central Cameroon. Agrofor. Syst. 88 (6): 983-1000.

Larzillière A, Vermeulen C, Dubiez E, Yamba Yamba T, Diowo S, Mumbere G. 2013. La maquette interactive, un outil novateur de participation. Bois For. Trop. 315 (1): 21-28.

Le Meur P-Y. 2002. Approche qualitative de la question foncière. Note méthodologique. Document de travail de l'Unité de Recherche 095, pp. 1-23. IRD REFO. 
Le Roy E. 1995. La sécurité foncière dans un contexte africain de marchandisation imparfaite de la terre. Terre, Terroir, Territoire. Les Tensions Foncières. Paris: ORSTOM.

Le Roy E, Karsenty A, Bertrand A. 1996. La sécurisation foncière en Afrique. Pour une gestion viable des ressources renouvelales. Paris: Karthala.

Maertens P, Rotmans J. 2005. Transitions in a globalising world. Futures 37: 1133-1144.

Magne A, Nonga N, Yemefack M, Robiglio V.2014. Profitability and implications of cocoa intensification on carbon emissions in Southern Cameroun. Agrof. Syst. 88 (6): 1133-1142.

Marien J-N, Louppe D, Dubiez E, Larzillière A. 2013. Quand la ville mange la forêt: les défis du bois énergie en Afrique centrale. France: Quae Éditions.

Ostrom E, Cox M. 2010. Moving beyond panaceas: a multi-tiered diagnostic approach for social-ecological analysis. Environ. Conserv. 37 (04): 451-463.

Oszwald J, Gond V, Tchiengué B, Nzigou Boucka F, Dallery D, Garcia C. 2015. Description des éléments paysagers des classifications d'occupation des sols CoForTips - Cameroun (CoForTips). Montpellier, France: CIRAD, p. 41.

Paul L, Simons G, Fennig C. 2015. Ethnologue: language of the World, 18th ed. Retrieved November 12, 2015, from https://www. ethnologue.com/language/yas

Pédelahore P. 2014. Systèmes agroforestiers à cacaoyers et transition capitaliste: l'exemple du Centre-Cameroun. Bois For. Trop. 321 (3).

Robinson C. 1983. Phonologie du gunu, parler yambassa : langue bantoue du Cameroun. Peeters Publishers.

Somwag G. 2014. Modélisation participative de la paysannerie agricole sur le territoire de Guéfigué et Guéboba. Toulouse: Université de Paule Sabatier.

Sonter L, Barrett D, Moran C, Soares-Filho B.2015. A land system meta-analysis suggests we underestmate intensive land uses in land change dynamics. J. Land Use Sci. 10 (2): 191-204.
Statistiques mondiales. 2015. Statistiques mondiales - Cameroun. Retrieved from http://www.statistiques-mondiales.com/

Toison V. 2008. Pratiques paysannes et aménagement forestier dans l'Est du Cameroun: quelle résilience socio-économique des systèmes locaux de gestion de ressources? Thèse. AgroParisTech, Paris.

Vermeulen C, Carrière S. 2001. Système foncier et forestier coutumier chez les Badjoués. In: La forêt des hommes : terroirs villageois en forêt tropicale africaine. Les presses agronomiques de Gembloux, Willy Delvingt, pp. 110-141.

Vermeulen C, Boldrini S, D'ans S, Schippers C. 2008. Maîtrises foncières et occupation de l'espace forestier dans le Nord-Est du Gabon. In: Les premières forêts communautaires du Gabon : récit d'une expérience pilote. Impribeau, Belgique: Vermeulen \& Doucet, pp. 15-26.

Vermeulen C, Dubiez E, Proces P, et al. 2011. Enjeux fonciers, exploitation des ressources naturelles et forêts des communautés locales en périphérie de Kinshasa, RDC. BASE Biotecnol. Agron. Soc. Environ. 15 (4): 535-544.

Vermeulen C, Meunier Q, Moumbogou C, Tiveau D, Bracke C. 2015. Les progrès de la foresterie sociale et communautaire. Presented at the XIV World Forestry Congress, Durban, South Africa.

Yambene Bomono H. 2010. Innovations paysannes en zone de contact forêt savane : le cas du pays Yambassa au Cameroun. Revue Internationale Des Sciences Humaines et Sociales 3 (3): 117-138.

Yambene Bomono H. 2012a. Course à la terre et dynamique des agroforêts en zone de contact forêt-savane (pays Yambassa au Cameroun). Revue Internationale Des Sciences Humaines et Sociales 5 (5): 247-258.

Yambene Bomono H. 2012b. Représentations et dynamiques foncières en zone de contact forêt-savane (pays yambassa) au Cameroun. Thèse de doctorat. Université de Paris I et Université de Ngaoundéré, Paris.

Citation de l'article : Gillet P, Codina Llavina E, Yambene H, Vermeulen C. 2016. Comment les villageois nomment-ils et s'approprient-ils leurs espaces ressources? Description d'un socio-écosystème en pays Yambassa, Cameroun. Cah. Agric. 25: 45006. 